\title{
Hyposmia Is Associated with Reduced Cognitive Function in COVID-19: First Preliminary Results
}

\author{
Agnes Pirker-Kees ${ }^{a, b}$ Kirsten Platho-Elwischger ${ }^{a}$ Sebastian Hafner ${ }^{a}$ \\ Kurt Redlich ${ }^{c}$ Christoph Baumgartner ${ }^{a, b}, d$

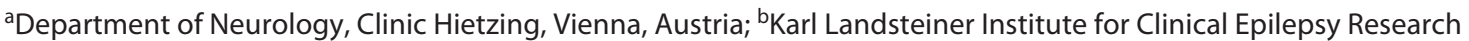 \\ and Cognitive Neurology, Vienna, Austria; ' $D$ Department of Rheumatology, Klinik Hietzing, Vienna, Austria; ${ }^{\mathrm{d} M e d i c a l}$ \\ Faculty, Sigmund Freud University, Vienna, Austria
}

\section{Key words}

Coronavirus disease $2019 \cdot$ Severe acute respiratory distress syndrome coronavirus- $2 \cdot$ Hyposmia $\cdot$ Cognition

\begin{abstract}
Background: Hyposmia is frequently reported as an initial symptom in coronavirus disease 2019 (COVID-19). Objective: As hyposmia accompanies cognitive impairment in several neurological disorders, we aimed to study whether hyposmia represents a clinical biomarker for both neurological involvement and cognitive impairment in mild COVID-19. We aimed to study whether olfactory dysfunction (OD) represents a clinical biomarker for both neurological involvement and cognitive impairment in mild COVID-19. Methods: Formal olfactory testing using the Sniffin'Sticks ${ }^{\circledR}$ Screening test, neuropsychological assessment using the Montreal Cognitive Assessment (MoCA), and detailed neurological examination were performed in 7 COVID-19 patients with mild disease course and no history of olfactory or cognitive impairment, and 7 controls matched for age, sex, and education. Controls were initially admitted to a dedicated COVID-19 screening ward but tested negative by real-time PCR. Results: The number of correctly identified odors was significantly lower in COVID-19 than in controls $(6 \pm 3$, vs.
\end{abstract}

$10 \pm 1 p=0.028, r=0.58$ ). Total MoCA score was significantly lower in COVID-19 patients than in controls ( $20 \pm 5$ vs. 26 $\pm 3, p=0.042, r=0.54)$. Cognitive performance indicated by MoCA was associated with number of correctly identified odors in COVID-19 patients and controls (COVID-19: $p=$ $0.018,95 \% \mathrm{Cl}=9-19$; controls: $p=0.18, r=0.63,95 \% \mathrm{Cl}=$ 13-18.5 $r=0.64)$. Discussion/Conclusion: OD is associated with cognitive impairment in controls and mild COVID-19. OD may represent a potentially useful clinical biomarker for subtle and even subclinical neurological involvement in severe acute respiratory distress syndrome coronavirus- 2 infection.

(c) 2021 S. Karger AG, Base

\section{Introduction}

Olfactory dysfunction (OD) is increasingly reported as a frequent initial symptom in mild to severe coronavirus disease 2019 (COVID-19) that arises from the severe acute respiratory distress syndrome coronavirus-2 (SARS-CoV-2) $[1,2]$. OD can be considered as a welldocumented clinical biomarker for cognitive impairment in several neurodegenerative disorders [3, 4]. In mild cognitive impairment, OD was associated with a faster rate
Correspondence to:

Agnes Pirker-Kees, agnes.pirker-kees@gmx.at 
of cognitive decline and might be predictive for progression to dementia [4]. In Parkinson's disease, OD is a wellknown premotor symptom [5], associated with dementia regardless of baseline cognitive function $[6,7]$. The olfactory mucosa represents a unique interface between the nervous and the immune system because olfactory receptor cells can transport various pathogens to the brain [8]. OD is present in $85 \%$ of moderate COVID-19 [2] and $98 \%$ of overall patients $[1,9]$. Compared to influenza-like symptoms, patients with OD were ten times more likely to have COVID-19 [10]. The occurrence of OD as an early symptom of cognitive dysfunction in several neurodegenerative diseases $[3,4]$ raises the question whether such an association also exists in COVID-19 and whether OD could be an early biomarker of subtle central nervous system (CNS) involvement in COVID-19.

Indeed, neurological symptoms, such as headache, alterations of consciousness, delirium, vertigo, seizures, or ataxia, have been reported in $36 \%$ (unselected) up to $84 \%$ (severely affected) COVID-19 patients $[11,12]$. In mild infection, a high prevalence of headache (70\%), visual disturbances, vertigo, nerve pain, ataxia, or fatigue was also reported [13]. CNS involvement might emerge within the context of severe COVID-19: ischemic stroke, sinus vein thrombosis, encephalitis, transverse myelitis, or encephalopathy is reported $[11,14,15]$. Besides the occurrence of pronounced neurological symptoms in severely affected COVID-19 patients, subtle alterations of cognitive functions in mild COVID-19 infections have not been investigated systematically, to the best of our knowledge.

Despite initially mild symptoms, the condition of some patients can suddenly deteriorate dramatically with the need for prolonged mechanical ventilation [16]. One possible explanation for this unfavorable clinical course could be CNS involvement. Indeed, transsynaptic transfer of various coronaviruses is well documented, and antigens of the other coronaviruses have been detected in the nucleus ambiguous and the nucleus of the solitary tract, which belong to the cardiorespiratory center [1719]. SARS-CoV-2 virus RNA has been isolated in the cerebrospinal fluid of one patient [15]. Early identification of patients with subtle neurological and neurocognitive symptoms could be crucial for further patient management and appropriate resource allocation.

We systematically studied the association of olfactory function, cognitive performance, and neurological symptoms in COVID-19 patients. We hypothesized that olfactory functions and cognitive functions were impaired in COVID-19 as compared to non-COVID-19 patients.

\section{Materials and Methods}

\section{Patients}

The study was conducted at the Clinic Hietzing, Vienna, Austria. Seven patients with confirmed SARS-CoV-2 infection admitted to a general ward, and 7 controls were included. Controls were selected among all patients, who were admitted to a neurological ward dedicated for COVID-19 screening, and then transferred to a general neurological ward after COVID-19 was ruled out. Based on age, sex, and years of education, controls were selected for each COVID-19 patient on a 1:1 basis. For patient characteristics, see Table 1. Inclusion criteria were i) $18-99$ years and ii) able to perform olfactory and cognitive assessment. Exclusion criteria were i) not able to perform olfactory and cognitive assessment, ii) history of or present ear-nose-throat disease with preexisting and/or current hyposmia, and iii) history of or present neurological disease with known alterations of olfactory sense or cognitive decline (e.g., dementia, Parkinson's disease, and frontal lobe lesions). None of the patients was admitted to an intensive care unit (ICU) during disease course, none had oxygen $\left(\mathrm{O}_{2}\right)$ supply, and patients were investigated between days 8 and 22 after diagnosis of COVID-19.

\section{Neurological Examination}

Neurological examination was performed by an experienced neurologist. Patients were asked for any neurological complains since COVID-19. Findings on physical examination were classified into meningism, cranial nerve abnormalities, brainstem signs, pyramidal signs, coordination abnormalities, sensory signs, and gait abnormalities.

\section{Olfactory Assessment}

To get an impression, if participants were aware of their OD, they were asked to semi-quantify their subjective olfactory function in general prior to the infection (worse than others, normal, or better than others) and their subjective alterations of olfactory sense at beginning of the actual disease (1: no change of olfactory function and 5: severe alteration of olfactory function/anosmia). Olfactory function was assessed using Sniffin'Sticks ${ }^{\circledR}$ (www.smelltest.eu), a widely used olfactory test battery in Europe [20], consisting of 12 pen-shaped sticks with different odors (orange, leather, cinnamon, peppermint, banana, lemon, liquorice, coffee, cloves, pineapple, rose, and fish) to be identified.

\section{Cognitive Assessment}

The Montreal Cognitive Assessment (MoCA) was performed to assess cognitive functions. This test assesses the domains attention and concentration, executive functions, memory, language, visuoconstructional skills, conceptual thinking, calculations, and orientation. A maximum of 30 points can be achieved; scores above 23-25 are considered normal, depending on age and education $[21,22]$.

\section{Statistical Analysis}

For statistical analysis, SPSS version 26 was used. Data were analyzed as seven case-control matched pairs. Due to small samples, nonparametric tests for paired samples were used: Wilcoxon sign-rank was used for discrete and categorical variables, and effect size was estimated as $\mathrm{r}$ ( $r>0.5$ indicates a strong effect). $\mathrm{McNe}$ mar's test was used for dichotomous variables. $p<0.05$ was considered statistically significant. 
Table 1. Baseline characteristics and neurological findings of COVID-19 patients and non-COVID-19 controls

\begin{tabular}{|c|c|c|c|c|c|c|c|}
\hline Pat ID & $\begin{array}{l}\text { Age, } \\
\text { years }\end{array}$ & Sex & $\begin{array}{l}\text { Days since } \\
\text { infection }\end{array}$ & $\begin{array}{l}\text { Days in } \\
\text { ICU }\end{array}$ & $\begin{array}{l}\text { Years of } \\
\text { education }\end{array}$ & $\begin{array}{l}\text { Neurological } \\
\text { complaints }\end{array}$ & $\begin{array}{l}\text { Neurological } \\
\text { examination }\end{array}$ \\
\hline \multicolumn{8}{|c|}{ COVID-19 patients } \\
\hline $\mathrm{C} 1$ & 70 & M & 21 & 0 & 18 & Visual hallucinations & Normal \\
\hline $\mathrm{C} 2$ & 78 & M & 8 & 0 & 8 & Headache & Normal \\
\hline $\mathrm{C} 3$ & 79 & M & 20 & 0 & 8 & None & Normal \\
\hline $\mathrm{C} 4$ & 73 & M & 22 & 0 & 12 & None & Normal \\
\hline C6 & 76 & $\mathrm{~F}$ & 19 & 0 & 8 & $\begin{array}{l}\text { Sensory disturbance lower } \\
\text { extremities }\end{array}$ & $\begin{array}{l}\text { Reflex-attenuation } \\
\text { Lower extremities }\end{array}$ \\
\hline $\mathrm{C} 7$ & 82 & $\mathrm{~F}$ & 9 & 0 & 8 & Headache & Normal \\
\hline \multicolumn{8}{|c|}{ Controls } \\
\hline $\mathrm{H} 1$ & 77 & M & & & 18 & Headache & Normal \\
\hline $\mathrm{H} 2$ & 71 & M & & 18 & Headache & Normal & \\
\hline H7 & 92 & $\mathrm{~F}$ & & 8 & none & Normal & \\
\hline
\end{tabular}

COVID-19, coronavirus disease 2019; ICU, intensive care unit.

\section{Results}

The study population consisted of 7 COVID-19 patients ( 3 females) and 7 controls ( 3 females). Patients and controls did not differ significantly regarding age (patients $79 \pm 8$ vs. controls $80 \pm 7, p>0.05$ ), sex, and years of education (patients $11 \pm 4$ vs. controls $12 \pm 5, p>0.05$ ). COVID-19 was mild in all patients, and none of them had to be admitted to an ICU during disease course. For baseline characteristics, see Table 1 . All patients and 6/7 controls rated their subjective olfactory function in general as normal or excellent. At the time of evaluation, olfactory sense was self-reported to be reduced in 6/7 (85\%) of COVID-19 patients (hyposmia in 4 and anosmia in 2 patients) and as usual in controls. When tested systematically using Sniffin'Sticks ${ }^{\circledR}$, hyposmia was present in $6 / 7$ COVID-19 patients (moderate $28 \%$, severe $42 \%$, and anosmia 14\%), whereas mild hyposmia was present in $1 / 7$ of controls (14\%).

The number of correctly identified odors was significantly lower in COVID-19 patients than in controls (6 \pm 3 , vs. $10 \pm 1 p=0.028, r=0.58$ ). One patient identified all odors correctly but reported a complete loss of gustatory sense since the infection with COVID-19.

In addition to the overall number of correctly identified odors, we were interested, if any particular odor was identified significantly less frequently depending on CO-
VID-19. Although not statistically significant, we want to mention the odors "lemon" and "clove" identified by $2 / 7$ (28\%) COVID-19 patients but 6/7 (85\%) controls.

None of the patients and controls reported about memory or other cognitive problems in the past, none had a known history of MCI or dementia, and none reported subjective cognitive impairment at the time of examination. Total MoCA score was significantly lower in COVID-19 patients than in controls $(20 \pm 5$ vs. $26 \pm 3$, $p=0.042, r=0,54)$. MoCA was indicative of MCI 6/7 COVID-19 patients (median 21/30, range 10-27) and in $1 / 7$ controls (median 25/30, range 21-30).

None of the MoCA subtests and domains was significantly different between patients and controls.

MoCA was directly associated with number of correctly identified odors in COVID-19 patients and controls (COVID-19: $p=0.018,95 \% \mathrm{CI}=9-19, r=0.63$; controls: $p=0.18,95 \% \mathrm{CI}=13-18.5, r=0.64)$. Neurological history revealed headache in 2/7 COVID-19 patients and in $1 / 7$ controls. None reported vertigo or dizziness. One highly educated man with COVID-19 without any psychiatric history reported visual hallucinations from day 18 (colored waves and flowers, occurring when eyes opened and closed, no association to circadian rhythm). He was fully communicable, orientated, and presented no symptoms of delirium. One female patient reported onset of sensory disturbances in her legs since day 15 after in- 
fection, vibration sense was $6 / 8$, and deep tendon reflexes were slightly attenuated. Lumbar puncture was not performed because of mild symptoms only. Headache was present in 2 of the controls and lower back pain in one control. None of the other patients or controls had neurological abnormalities on clinical neurological examination. Frequency of any symptom did not differ significantly between patients and controls.

\section{Discussion/Conclusion}

This appears to be the first study demonstrating an association of olfactory and cognitive dysfunction in mild COVID-19. Cognitive dysfunction was documented in association with OD in all but one COVID-19 patient, and in only one individual in the control group, despite no one had a history of dementia or subjective memory complaints.

These findings are in line with our study hypothesis. It is well known that the olfactory system represents a key entry port for pathogens including viruses and environmental toxins to the CNS [4]. OD is known as occasional presymptomatic sign of various neuroimmunological and neurodegenerative disorders [8], and it is predictive of cognitive decline in mild cognitive impairment, Alzheimer' disease, Parkinson's disease [7], multiple sclerosis [23], and in dementia-free older adults [4]. The possible contribution of inflammation and viral infections (e.g., human herpesviruses and cytomegalovirus) in the pathogenesis of mild cognitive impairment and Alzheimer's disease is worth mentioning [24, 25], but given the high prevalence of those viruses, the meaning of these epidemiological data is difficult to interpret. Because of the recency of COVID-19 pandemic, the association of OD and cognitive dysfunction in COVID-19 could serve as an epidemiological and pathophysiological model, in terms of the olfactory system as a gate to the CNS, and the association of viral infections with neurodegenerative and neuroinflammatory diseases.

However, there is no systematic data about cognitive performance in the course of COVID-19. It is well described that neurological symptoms in moderate to severe COVID-19 include headache (14\%), impaired consciousness $(7.5 \%$ up to $22 \%)$, delirium/agitation $(69 \%)$, confusion (10\%), and seldom seizures $[11,12,14,16,19$, 26]. Some patients can suddenly develop severe encephalopathy $[11,15]$. In contrast, data about neurological involvement in the mild course are limited, to reports of higher prevalence of headache (70\%), vertigo, dizziness, or fatigue [13]. However, OD is a typical and well-described feature in COVID-19 [10]. It is reported in up to $85 \%$ of mild [2] and $98 \%$ of severe COVID-19 [1].

In the light of our novel findings, cognitive dysfunction and OD in mild COVID-19 might represent one end of a spectrum of subtle CNS involvement - with the olfactory system as the entry port. Delirium, confusion, and alterations of consciousness in severe COVID-19 might represent the other end of the spectrum with severe CNS involvement. However, the exact pathophysiological mechanism remains speculative, and the long-term cognitive outcome after subtle or severe CNS involvement in COVID-19 cannot yet be predicted.

In terms of OD, post-viral anosmia has been reported in previous studies $[9,27,28]$. SARS-CoV-2 has the ability to enter epithelial cells, depending on their expression of angiotensin converting receptor 2 and the TMPRSS2protease. The nasal epithelial cells and the olfactory bulb [29] showed the highest expression and therefore are the main gate for SARS-CoV-2 entrance [30]. Viral invasion of those regions is supposed to be responsible for OD in COVID-19 [29].

In terms of CNS involvement and possible cognitive decline, angiotensin converting receptor 2 is also expressed on astrocytes, oligodendrocytes, and neurons [31], which might bear the potential to promote CNS involvement. This is in accordance with increasing reports of meningoencephalitis [15], myelitis [32], or encephalopathy $[14,16]$ leading to alterations of consciousness or delirium in severe COVID-19 [33].

Several limitations of our study need to be mentioned. The number of patients in our study was rather small, but sample size was limited due to strict hygienic measures in the hospital. A confirmation of our significant results in a larger number of patients is needed. Even though patients negotiated cognitive problems, we cannot exclude slight subclinical cognitive impairment prior to SARS$\mathrm{CoV}-2$ infection. Structural brain imaging is not available because it was not indicated during hospital stay. $\mathrm{O}_{2}$ saturation can be a long-term risk factor for cognitive decline [34] but was not measured at time of investigation. All patients were on a normal ward, and no one needed $\mathrm{O}_{2}$ supply or had objective signs of dyspnea. There was no indication for monitoring the $\mathrm{O}_{2}$ saturation. Silent hypoxia cannot be ruled out as a potential cofactor for cognitive impairment. This might be an important aspect to consider in future studies. Patients were included in the study between days 8 and 22 after diagnosis, according to the inclusion criteria of mild COVID-19, no ICU stay, and clinically stable. Hyposmia persists least 7 days in 
COVID-19 [2]. All but one of our patients had OD, so we would not expect this time frame to affect our results.

In conclusion, we documented cognitive dysfunction in association with OD in mild to moderate affected COVID-19 patients and an association of olfactory and cognitive dysfunction in these individuals. OD thus might be considered as a clinical biomarker for CNS involvement not apparent on routine clinical assessment. While olfactory impairment due to viral infection is mostly reversible [28], the long-term course of cognitive dysfunction in COVID-19 needs to be studied systematically in the future and might contribute to the understanding of the role of the olfactory system as gatekeeper to the CNS.

\section{Statement of Ethics}

The study was approved by the local Ethics Committees "EthikKommission der Stadt Wien" according to the Helsinki Declaration of 1975, and all patients gave written informed consent to participate in the study.

\section{Conflict of Interest Statement}

The authors have no conflicts of interest to declare.

\section{Funding Sources}

No funding was raised for this study.

\section{Author Contributions}

Pirker-Kees A.: substantial contribution to the conception, data acquisition, analysis, drafting, final approval, and agreement to be accountable for all aspects of the work. Hafner S.: substantial contribution to the data acquisition, drafting, final approval, and agreement to be accountable for all aspects of the work. PlathoElwischger K.: substantial contribution to the conception, data acquisition, drafting, final approval, and agreement to be accountable for all aspects of the work. Redlich K.: substantial contribution to the data acquisition, drafting, final approval, and agreement to be accountable for all aspects of the work. Baumgartner C.: substantial contribution to the conception, analysis, revising it critically for important intellectual content, final approval, and agreement to be accountable for all aspects of the work.

\section{References}

1 Moein ST, Hashemian SMR, Mansourafshar B, Khorram-Tousi A, Tabarsi P, Doty RL. Smell dysfunction: a biomarker for COVID-19. Internat Forum Allergy Rhinol. 2020;10:944-50.

2 Lechien JR, Chiesa-Estomba CM, De Siati DR, Horoi M, Le Bon SD, Rodriguez A, et al. Olfactory and gustatory dysfunctions as a clinical presentation of mild-to-moderate forms of the coronavirus disease (COVID-19): a multicenter European study. Eur Arch Otorhinolaryngol. 2020 Aug;277(8):2251-61.

3 Churnin I, Qazi J, Fermin CR, Wilson JH, Payne SC, Mattos JL. Association between olfactory and gustatory dysfunction and cognition in older adults. Am J Rhinol Allergy. 2019 Mar;33(2):170-7.

4 Dintica CS, Marseglia A, Rizzuto D, Wang R, Seubert J, Arfanakis K, et al. Impaired olfaction is associated with cognitive decline and neurodegeneration in the brain. Neurology. 2019;92(7):e700-9.

5 Saini D, Mukherjee A, Roy A, Biswas A. A comparative study of the behavioral profile of the behavioral variant of frontotemporal dementia and Parkinson's disease dementia. Dement Geriatr Cogn Dis Extra. 2020 SepDec;10(3):182-94.

6 Domellof ME, Lundin KF, Edstrom M, Forsgren L. Olfactory dysfunction and dementia in newly diagnosed patients with Parkinson's disease. Parkinsonism Relat Disord. 2017 May;38:41-7.
7 Marin C, Vilas D, Langdon C, Alobid I, López-Chacón M, Haehner A, et al. Olfactory dysfunction in neurodegenerative diseases. Curr Allergy Asthma Rep. 2018 Jun 15;18(8): 42.

8 Shin T, Kim J, Ahn M, Moon C. Olfactory dysfunction in CNS neuroimmunological disorders: a review. Mol Neurobiol. 2019 May;56(5):3714-21.

9 Bagheri SHR, Asghari AM, Farhadi M, Shamshiri AR, Kabir A, Kamrava SK, et al. Coincidence of COVID-19 epidemic and olfactory dysfunction outbreak. medRxiv. 2020.

10 Yan $\mathrm{CH}$, Faraji F, Prajapati DP, Boone CE, DeConde AS. Association of chemosensory dysfunction and COVID-19 in patients presenting with influenza-like symptoms. Int Forum Allergy Rhinol. 2020;10(7):806-13.

11 Helms J, Kremer S, Merdji H, Clere-Jehl R, Schenck M, Kummerlen C, et al. Neurologic features in severe SARS-CoV-2 infection. N Engl J Med. 2020;382(23):2268-70.

12 Mao L, Jin H, Wang M, Hu Y, Chen S, He Q, et al. Neurologic manifestations of hospitalized patients with coronavirus disease 2019 in Wuhan, China. JAMA Neurol. 2020;77:68390.

13 Lechien JR, Chiesa-Estomba CM, Place S, Van Laethem Y, Cabaraux P, Mat Q, et al. Clinical and epidemiological characteristics of 1420 European patients with mild-to-moderate coronavirus disease 2019. J Intern Med. 2020 Sep;288(3):335-44.
14 Divani AA, Andalib S, Biller J, Di Napoli M, Moghimi N, Rubinos CA, et al. Central nervous system manifestations associated with COVID-19. Curr Neurol Neurosci Rep. 2020 October 30;20(12):60.

15 Moriguchi T, Harii N, Goto J, Harada D, Sugawara $\mathrm{H}$, Takamino J, et al. A first case of meningitis/encephalitis associated with SARS-Coronavirus-2. Int J Infect Dis. 2020 Apr 3;94:55-8.

16 Chen T, Wu D, Chen H, Yan W, Yang D, Chen G, et al. Clinical characteristics of 113 deceased patients with coronavirus disease 2019: retrospective study. Bmj. 2020 Mar 26; 368:m1091.

17 Hadziefendic S, Haxhiu MA. CNS innervation of vagal preganglionic neurons controlling peripheral airways: a transneuronal labeling study using pseudorabies virus. J Auton Nerv Syst. 1999 May 28;76(2-3):135-45.

18 Matsuda K, Park CH, Sunden Y, Kimura T, Ochiai $\mathrm{K}$, Kida $\mathrm{H}$, et al. The vagus nerve is one route of transneural invasion for intranasally inoculated influenza a virus in mice. Vet Pathol. 2004 Mar;41(2):101-7.

19 Li YC, Bai WZ, Hashikawa T. The neuroinvasive potential of SARS-CoV2 may play a role in the respiratory failure of COVID-19 patients. J Med Virol. 2020 Feb 27;92:55-5. 
20 Hummel T, Sekinger B, Wolf SR, Pauli E, Kobal G. 'Sniffin' sticks': olfactory performance assessed by the combined testing of odor identification, odor discrimination and olfactory threshold. Chem Senses. 1997 Feb;22(1): 39-52.

21 Tan JP, Li N, Gao J, Wang LN, Zhao YM, Yu BC, et al. Optimal cutoff scores for dementia and mild cognitive impairment of the Montreal Cognitive Assessment among elderly and oldest-old Chinese population. J Alzheimers Dis. 2015;43(4):1403-12.

22 Zhou Y, Ortiz F, Nuñez C, Elashoff D, Woo E, Apostolova LG, et al. Use of the MoCA in detecting early Alzheimer's disease in a spanishspeaking population with varied levels of education. Dement Geriatr Cogn Dis Extra. 2015; 5(1):85-95.

23 Atalar AÇ, Erdal Y, Tekin B, Yıldız M, Akdoğan Ö, Emre U. Olfactory dysfunction in multiple sclerosis. Mult Scler Relat Disord. 2018 Apr;21:92-6.
24 Pirker A, Schmied C, Dal-Bianco P. T-cells show increased production of cytokines and activation markers in Alzheimer's disease. Brain Disord. Ther. 2013;3(1):4.

25 Sochocka M, Zwolińska K, Leszek J. The infectious etiology of Alzheimer's disease. Curr Neuropharmacol. 2017;15(7):996-1009.

26 Chen N, Zhou M, Dong X, Qu J, Gong F, Han $\mathrm{Y}$, et al. Epidemiological and clinical characteristics of 99 cases of 2019 novel coronavirus pneumonia in Wuhan, China: a descriptive study. Lancet. 2020 Feb 15;395(10223):50713.

27 Welge-Lüssen A. Re-establishment of olfactory and taste functions. GMS Curr Top Otorhinolaryngol Head Neck Surg. 2005;4: Doc06.

28 Soler ZM, Patel ZM, Turner JH, Holbrook EH. A primer on viral-associated olfactory loss in the era of COVID-19. Int Forum Allergy Rhinol. 2020 Apr 9;10:814-20.

29 Sungnak W, Huang N, Bécavin C, Berg M, Queen R, Litvinukova M, et al. SARS-CoV-2 entry factors are highly expressed in nasal epithelial cells together with innate immune genes. Nat Med. 2020 April 23;26:681-7.
30 Butowt R, Bilinska K. SARS-CoV-2: olfaction, brain infection, and the urgent need for clinical samples allowing earlier virus detection. ACS Chem Neurosci. 2020 Apr 13;11:1200-3.

31 Chen R, Wang K, Yu J, Chen Z, Wen C, Xu Z. The spatial and cell-type distribution of SARS-CoV-2 receptor ACE2 in human and mouse brain. bioRxiv. 2020.

32 Sarma D, Bilello LA. A case report of acute transverse myelitis following novel coronavirus infection. Clin Pract Cases Emerg Med. 2020 Aug;4(3):321-3.

33 Pezzini A, Padovani A. Lifting the mask on neurological manifestations of COVID-19. Nat Rev Neurol. 2020 November1;16(11): 636-44.

34 Wang Y, Li X, Wei B, Tung TH, Tao P, Chien CW. Association between chronic obstructive pulmonary disease and dementia: systematic review and meta-analysis of cohort studies. Dement Geriatr Cogn Dis Extra. 2019 MayAug;9(2):250-9. 